\title{
Depth Measurement of Moving Slurry at the Wet End of a Paper Machine
}

\author{
James S. Goddard, Jr. ${ }^{\dagger a}$, Hamed Sari-Sarraf ${ }^{\text {a }}$, John C. Turner ${ }^{2}$, Martin A. Hunt ${ }^{\mathrm{a}}$, and \\ Besma R. Abidi ${ }^{\text {b }}$ \\ ${ }^{a}$ Oak Ridge National Laboratory ${ }^{\ddagger}$, P.O. Box 2008, Oak Ridge, Tennessee 37831-6011 \\ ${ }^{b}$ Dept. of Electrical Engineering, University of Tennessee, Knoxville, Tennessee
}

\begin{abstract}
The paper industry has long had a need to better understand and control its papermaking process upstream, specifically at the wet end in the forming section of a paper machine. A vision-based system is under development that addresses this need by automatically measuring and interpreting the pertinent paper web parameters at the wet end in real time. The wet-end characterization of the paper web by a vision system involves a four-dimensional measurement of the slurry in real time. These measurements include the two-dimensional spatial information, the intensity profile, and the depth profile. This paper describes the real-time depth profile measurement system for the high-speed moving slurry. A laser line-based measurement method is used with a high-speed programmable camera to directly measure slurry height. The camera is programmed with a profile algorithm, producing depth data at fast sampling rates. Analysis and experimentation have been conducted to optimize the system for the characteristics of the slurry and laser line image. On-line experimental results are presented.
\end{abstract}

Keywords: Paper, inspection, laser, profilometry, CMOS, range imaging

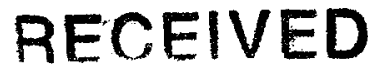

\section{INTRODUCTION}

MAR 031999

A number of on-line sensors, including vision systems, have been developed for measuring paper properties. ${ }^{1.2,5}$ However, nearly all are intended for the dry end and typically scan across the web resulting in less than $100 \%$ web coverage. A small number of wet-end sensors have been previously described. Niemi has used a camera and illumination to determine the location and shape of the dry line at the wet end of a Fourdrinier machine. ${ }^{3}$ Viewing the wet end with the aid of a strobe light as a diagnostic tool is a common practice in the industry. Generally, by observing the web in this form, nonuniformities, flocculation, and the table action of the slurry on the wire may be discerned. Nomura has used stroboscopic imaging to show the varying nonuniformities in the sheet due to variations in headbox design. ${ }^{6}$ The headbox flow conditions have been shown to directly affect fiber orientation and other formation properties. ${ }^{4,7}$ While stroboscopic imaging is established as a viable on-line technique for paper web sensing, structured lighting techniques have only been used in off-line applications, such as measuring the surface roughness of paper and board. ${ }^{2}$ In other application areas of computer vision, however, depth or range measurement using laser-based, structured lighting is a well-established method. ${ }^{9.10,11,12}$ A vision-based system is under development that automatically measures and interprets the pertinent paper web parameters at the wet end in real time. The wet-end characterization of the paper web by a vision system involves a four-dimensional measurement of the slurry in real time. These measurements include the two-dimensional spatial information in the machine direction and cross direction coordinates, the intensity profile, and the depth profile. The work to date has concentrated on the development of two subsystems to acquire these measurements. The first, the stroboscopic subsystem, acquires intensity images of the web using a CCD camera and a strobe light to freeze motion. The second is the depth-profiling subsystem whereby the depth profile of the slurry is measured in real time using a

\footnotetext{
$\dagger$ J.S.G. (Correspondence) Email: goddardjsjr@ornl.gov; Address: Oak Ridge National Laboratory, P.O. Box 2008, Oak Ridge, Tennessee; 37831-6011; Telephone: 423-574-9034; Fax: 423-574-6663.

₹ Research sponsored by the DOE Office of Industrial Technologies. OAK RIDGE NATIONAL LABORATORY is managed by LOCKHEED MARTIN ENERGY RESEARCH CORPORATION for the U.S. DEPARTMENT OF ENERGY under contract DE-AC05-960R22464.
} 


\section{DISCLAIMER}

This report was prepared as an account of work sponsored by an agency of the United States Government. Neither the United States Government nor any agency thereof, nor any of their employees, make any warranty, express or implied, or assumes any legal liability or responsibility for the accuracy, completeness, or usefulness of any information, apparatus, product, or process disclosed, or represents that its use would not infringe privately owned rights. Reference herein to any specific commercial product, process, or service by trade name, trademark, manufacturer, or otherwise does not necessarily constitute or imply its endorsement, recommendation, or favoring by the United States Government or any agency thereof. The views and opinions of authors expressed herein do not necessarily state or reflect those of the United States Government or any agency thereof. 


\section{DISCLAIMER}

Portions of this document may be illegible in electronic image products. Images are produced from the best available original document. 
- CMOS camera and structured lighting. This project extends the use of strobe lighting techniques to real-time $100 \%$ web measurements with sophisticated image processing approaches to extract the desired information. The addition of depth profile measurement in real time provides a new approach to investigating the formation properties. This paper describes the real-time depth profile measurement system for the high speed moving slurry. A laser linebased measurement method is used with a high-speed programmable camera to directly measure slurry height. Image analysis techniques are employed to extract those parameters that correlate with essential paper web properties which affect production yield and product quality. The following describes the technical approach used in the design and development of the depth-profiling subsystem and provides the results obtained from testing.

\section{DEPTH PROFILE MEASUREMENT}

The depth profile measurement system measures the surface profile of the wet-end slurry of a paper machine. The measurement is in the cross-direction of the slurry at a location downstream from the headbox. The headbox on a paper machine produces a jet of slurry consisting of mostly water with a small percentage of wood fiber and other materials. The jet is directed over a moving "wire" that carries the slurry over successive blades in which water is removed until the web can be taken up by rollers and then carried to multiple drying stages. The immediate area downstream from the headbox is called the table area. In this area, the primary formation properties of the finished paper are set. The slurry in this area is turbulent with random moving patterns along with a more regular pattern of small waves that tend to persist through the length of the table. The more random patterns introduce inhomogeneities into the slurry that may appear and disappear or may continue through to the dry area. Since the slurry has motion in depth as well as length and width, the acquisition and analysis of the depth profile is necessary to characterize the slurry shape and properties.

Structured lighting is employed through the use of an infrared laser for illumination and a special purpose CMOS camera to capture images of the laser profile. Figure 1 shows a picture of the system in which the laser is mounted vertically above the slurry while the camera views the scene from the side at approximately a 45 degree angle. The laser projects a line onto the slurry in the cross-direction. A high-speed CMOS camera with built-in processing capability processes the image data illuminated by the laser and determines the profile in terms of the height of the slurry. The geometry is shown in Figure 2 . For this case, with a vertical projection plane, the depth of the slurry as seen by the camera is given by,

$$
\Delta r=\Delta s \frac{B+f \cos \alpha}{f \cos ^{2} \alpha}
$$

where, $\alpha$ is the angle of the camera's optical axis from the horizontal, $\Delta r$ is the change in depth, $\Delta s$ is the change in the sensor location, $f$ is lens focal length, and $B$ is the baseline from the camera optical center to the laser. The camera is oriented so that the horizontal, or $x$ direction, is in the cross direction while the vertical, or $y$ direction, corresponds to the height measurement.

The depth profiling system was initially developed and tested in the laboratory to demonstrate feasibility. A standard RS170 camera can acquire the laser line images, but the speed is limited to 30 frames per second and the entire image must be processed. With the addition of the CMOS camera, a significant increase in the number of profiles per second is realized. The camera has a 256 by 256 pixel CMOS area array chip with 256 one-bit processors on chip. The processors are programmable and can process the images at high frame rates. Rates are dependent on the algorithm used to detect the laser line in the image and range from 100 to 900 profiles per second. Table 1 shows a list of the hardware used in the system. The infrared pass filter is used to exclude visible light so that external lighting does not interfere with the algorithm used to locate the laser line position. The linear polarizer further reduces any remaining interference by removing cross-polarized components such as specular reflections. 
Table 1. List of Hardware Used In Depth Profile Measurement System

\begin{tabular}{|l|l|}
\hline Camera & IVP Ranger with MAPP chip \\
\hline Lens & $25,30,50,75 \mathrm{~mm}$. focal length C-mount \\
\hline Infrared Pass filter & \\
\hline Computer system & $\begin{array}{l}\text { IBM compatible PC, 90 MHz, Pentium with IVP PCI } \\
\text { interface to camera }\end{array}$ \\
\hline Illumination & $\begin{array}{l}\text { Lasiris } 100 \mathrm{mw} ., 830 \mathrm{~nm} ., \text { infrared diode laser with } 45 \\
\text { degree fanout line projector }\end{array}$ \\
\hline Polarizing filter & Schneider screw-on mount linear polarizer \\
\hline
\end{tabular}

An infrared laser at $830 \mathrm{~nm}$. wavelength is invisible to the eye, but the silicon in the camera is highly sensitive to energy at this wavelength. The Lasiris laser projects a single line of light onto the surface at a fanout of 45 degrees. For a three feet line width at the surface, the laser height above the surface is about 3.6 feet. The baseline separation of the camera and the laser is approximately the same.

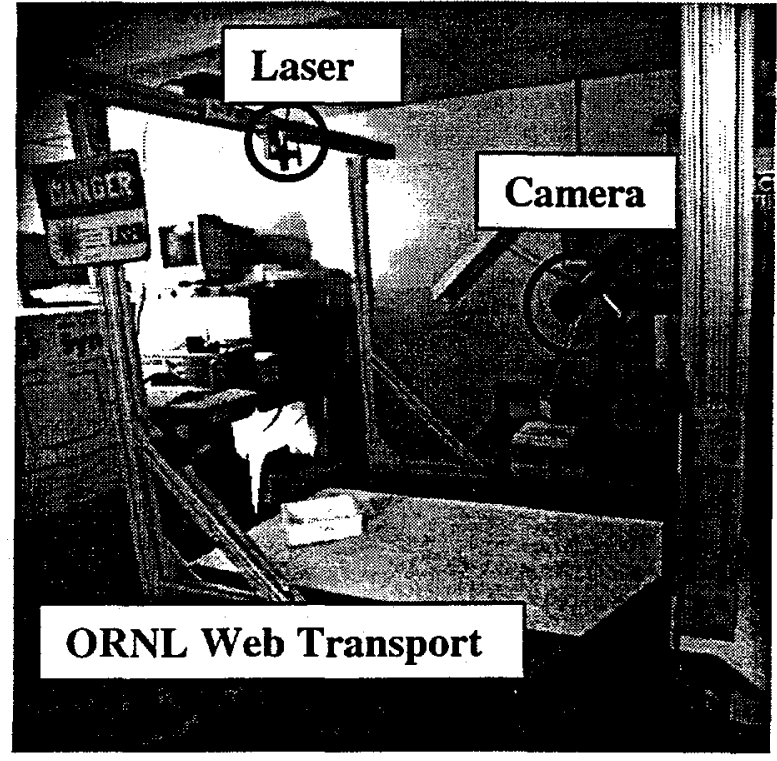

Figure 1. Picture of laser and camera mounted on ORNL test bed.

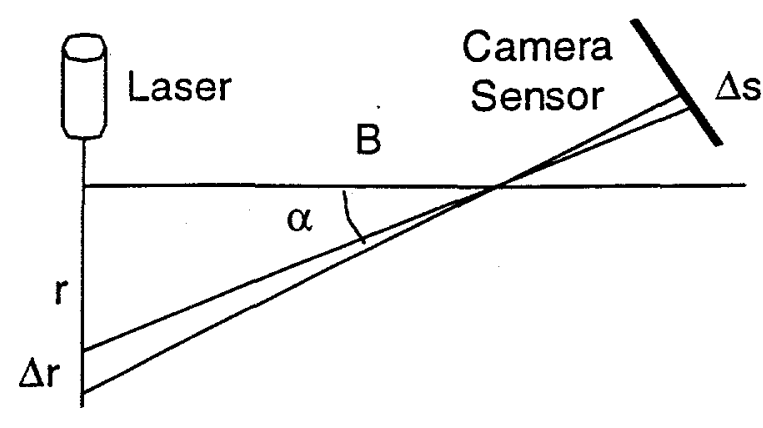

Figure 2. Diagram of laser and camera geometry.

During operation, the laser sheet of light intersects the surface of the moving slurry producing an irregular line. The camera acquires an image of this line, locates the line based on the selected algorithm, and transfers the profile data to the PC for analysis and display. Over time a two-dimensional image is assembled in the PC resulting in the time profile at that web location as well as the cross-direction profile.

Two laser line-finding algorithms have been evaluated with the CMOS camera. These algorithms were considered for robustness in the presence of noise and for speed of execution on the sensor. The first, a binary threshold method, applies a single threshold to the laser line intensity at each column of the image. If the intensity is found to be above the threshold at columns $x_{1}$ and $x_{2}$, the position of the line is taken to be $l=\frac{x_{1}+x_{2}}{2}$. For a symmetrical intensity profile with no noise, this method gives a good estimate of the center of the laser line, producing resolutions of one-half pixel. However, Johannesson ${ }^{12}$ has shown that with moderate noise, significant errors can be present. The second method is a maximum-finding algorithm using successive approximation. Successive thresholds are applied until the threshold closest to but less than the maximum is found. Then the position is 
determined-using the binary threshold formula given above. This method is more robust with significantly lower errors in the presence of noise than the binary threshold method. ${ }^{12}$ As expected, however, speed of execution is slower on the MAPP chip due to the application of multiple thresholds.

An enhancement to the basic algorithms has been added to measure multiple laser lines in a single image. A multiple line measurement is desirable on the slurry to provide a spatial distribution of the profile in the machine direction in addition to the cross-direction. This capability enables measurement of the time behavior of the profiles both on and between the table blades. Initially, the lines in the image must be found. Then the basic algorithm is applied to each of the lines.

\section{TEST RESULTS}

The depth measurement system was developed and tested in the laboratory as shown in Figure 1. Testing has subsequently been conducted on a Fourdrinier paper machine. A mount was built to properly position the camera and laser over the slurry about 10 feet downstream from the headbox and about 5 feet in from the side. Figure 3 shows an intensity image of the slurry where the machine direction is from left to right. As indicated previously, relatively large structures are present that can persist over time causing formation problems in the finished paper. The structures seen here have variations in intensity primarily due to shadowing. These are actually variations in . depth and provide the rationale for acquiring and analyzing the depth data.

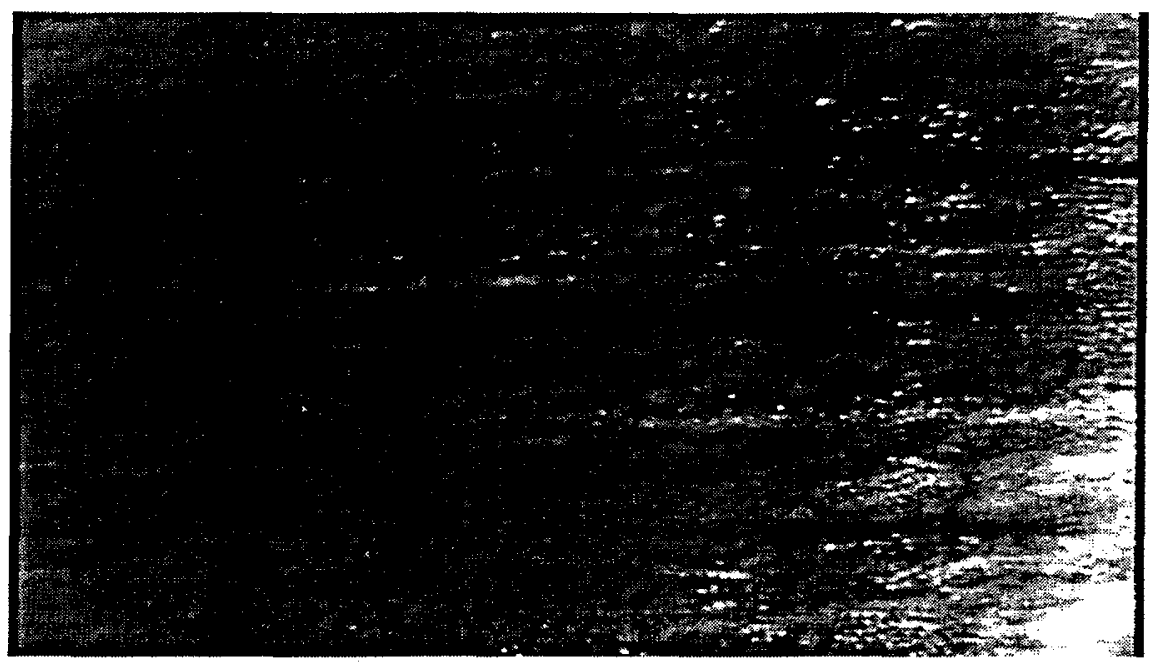

Figure 3. Intensity image of moving paper slurry taken with an area camera and strobe light.

Figure 4 is an intensity image of the laser line in the slurry. Note that the edges of the laser line are not sharp or well defined but appear to have a gradual falloff in intensity. The somewhat diffused illumination spreads from the center of the line. This behavior is due to the laser illumination penetrating into the slurry causing a gradual falloff in intensity instead of a direct reflection off the top surface of the slurry. Figure 5 shows three cross-section profiles of the laser line intensity from Figure 4. This figure confirms that the intensity gradually falls off on either side of the peak. The profile is seen to be asymmetrical as well. This result indicates that the binary threshold algorithm would not be an accurate predictor of line location. The real-time profile measurement confirmed this prediction. Although high profile rates were obtained, the binary threshold method was found to give poor results with highly inaccurate positions and numerous dropouts. In contrast, the successive approximation method gave consistent and accurate measurements with few dropouts. The successive approximation method provided a rate of 100 profiles/sec. compared to 600 profiles/sec. for the binary threshold algorithm. 


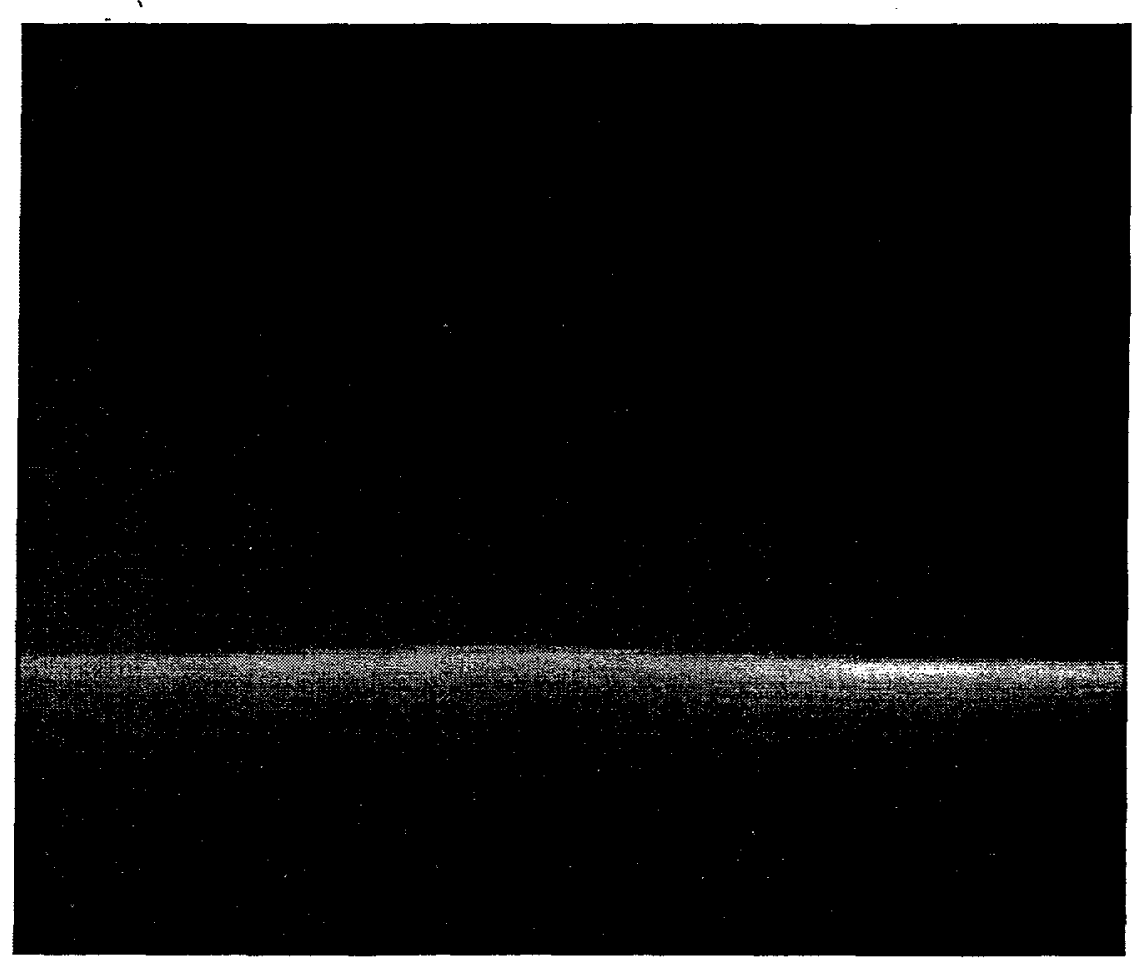

Figure 4. Intensity image of laser line on moving paper slurry. The intensity shows the diffusion of the light into the slurry resulting in a line with poorly defined edges.

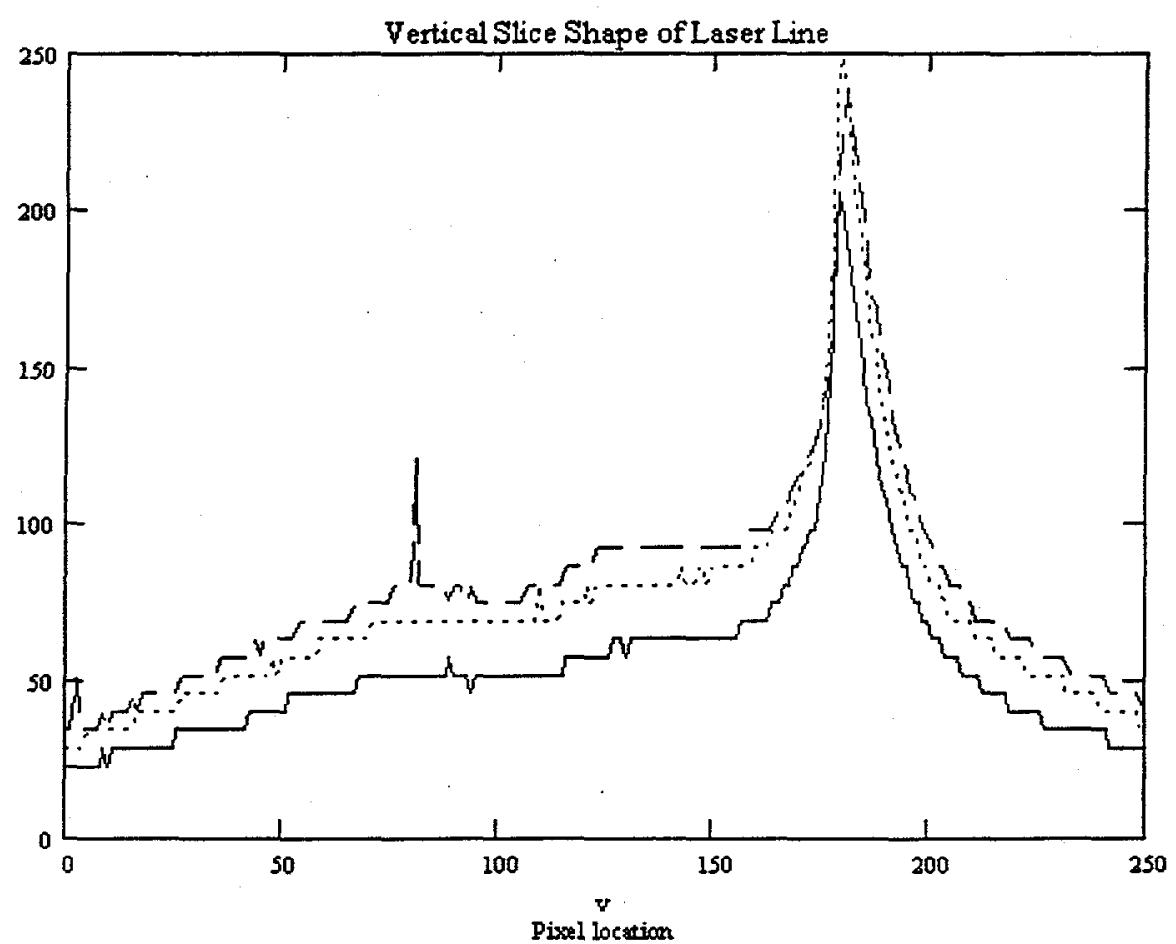

Figure 5. Intensity slice of laser line in Figure 4 at three different locations. The intensity is seen to be asymmetrical and to fall off gradually from the peak. 
Figures 6, 7, and 8 are the results of the profile measurements for focal lengths of 30,50 , and $75 \mathrm{~mm}$. respectively. Widths in the cross-direction are $10 \mathrm{in} ., 6 \mathrm{in}$, and $4 \mathrm{in}$. With a web speed of $1200 \mathrm{ft} . / \mathrm{min}$., the distance between samples is about $2.4 \mathrm{in}$. The wave profile of the slurry is clear from the graphs. Also note that the signal or range of values is greater for the larger focal lengths. However, the tradeoff is that the camera sees a smaller view.

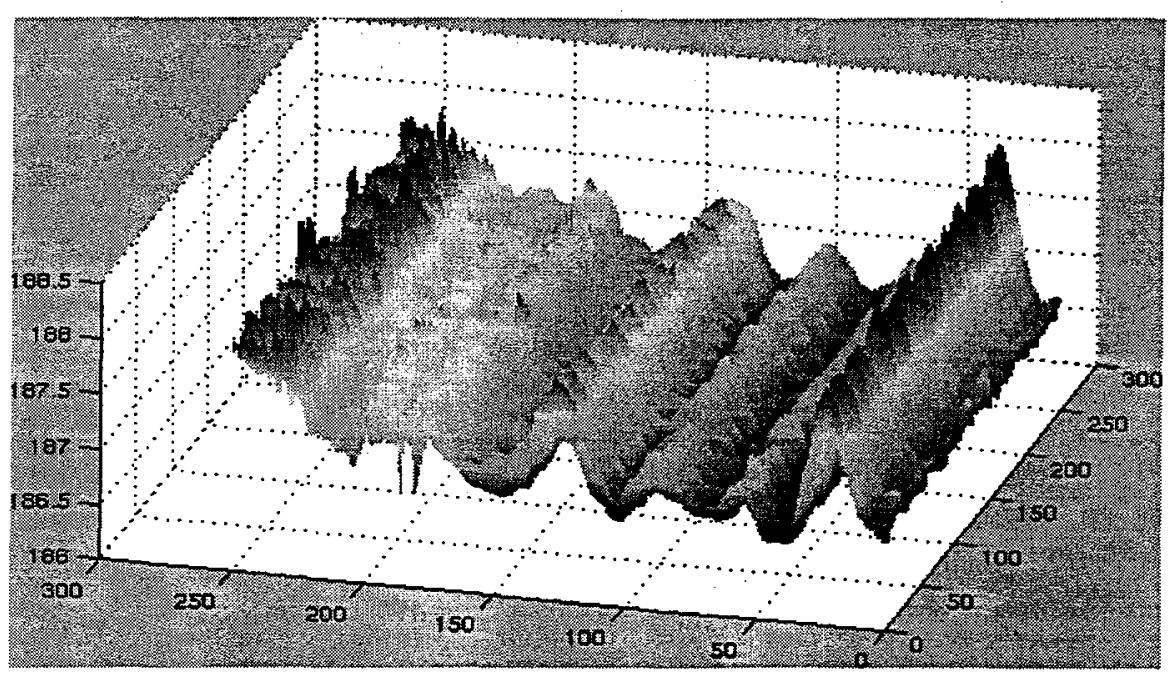

Figure 6. Range plot of depth profile measurement using focal length of $30 \mathrm{~mm}$.

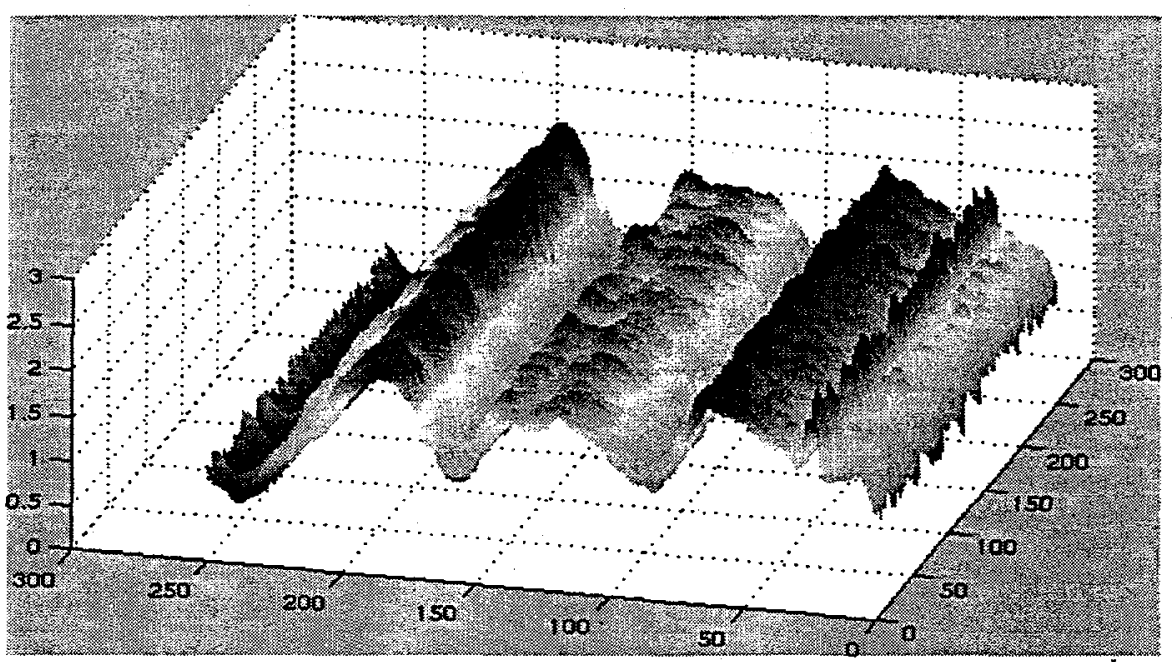

Figure 7. Range plot of depth profile measurement using focal length of $50 \mathrm{~mm}$. 


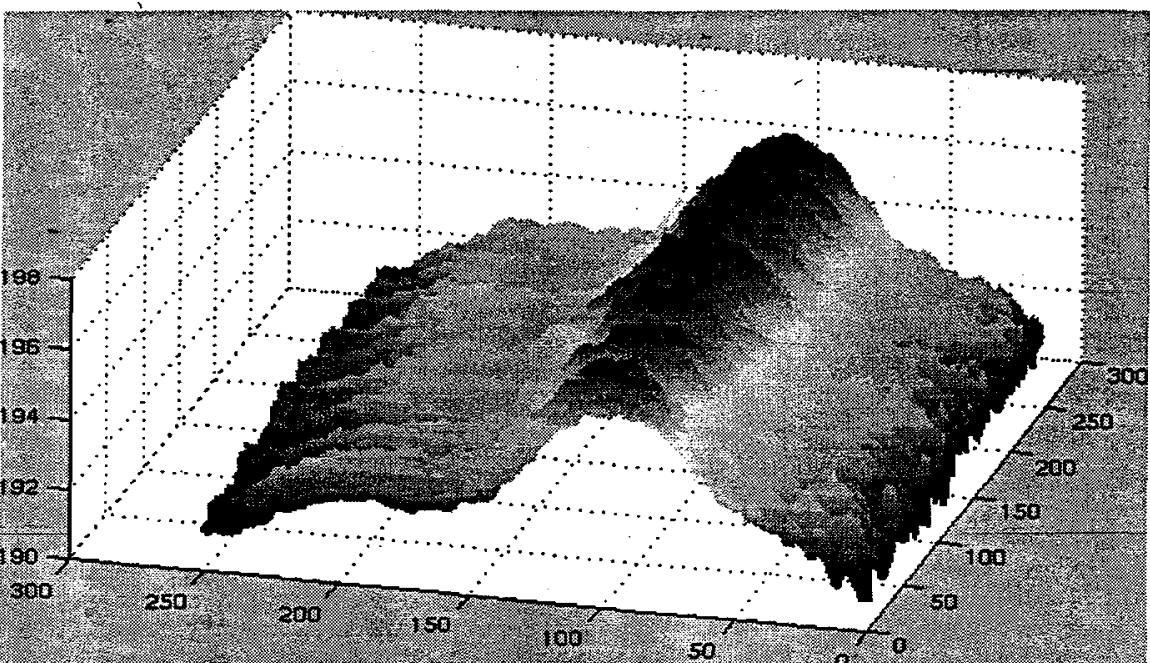

Figure 8. Range plot of depth profile measurement using focal length of $70 \mathrm{~mm}$.

\section{CONCLUSION}

A system has been developed for measuring the depth profile of the moving slurry at the wet end of a paper machine. This system is vision-based and uses structured light along with a high-speed CMOS sensor-based camera to acquire profiles of the moving slurry in real time. Since the algorithm for measuring the profile is executed on the CMOS chip, high profile rates are obtained. Actual in-plant testing on a paper machine has been conducted with good results. The system has been shown to acquire profiles of the slurry with resolution depending on the magnification. Improvements such as an anamorphic lens would permit high magnifications in the depth while maintaining a wide field of view. Spatial variation of the slurry can be measured by projecting multiple laser lines instead of a single line.

\section{REFERENCES}

1. K. Humphrey, Image Analysis, Pira International, 1995.

2. S. I'Anson, "3D laser surface profiling of paper and board," Paper Technology 38, No. 2, pp. 43-49, March 1997.

3. A. J. Niemi and C. J. Backstrom, "Automatic observation of dry line on wire for wet end control of the paper machine," Pulp and Paper Canada 95, No. 2, pp. 27-30, February 1994.

4. C. K. Aidun, "Hydrodynamics of streaks on the forming table," Tappi Journal, August 1997.

5. J-P. Bernie and W.J.M. Douglas, "Local grammage distribution and formation of paper by light transmission image analysis," Tappi Journal 79, No. 1, pp. 193-202, January 1996.

6. T. Nomura, K. Wada and T. Shimizu, "High consistency sheet forming," Tappi Journal, pp. 115-122, January 1989.

7. C. K. Aidun and A. E. Kovacs, "Hydrodynamics of the forming section: the origin of nonuniform fiber orientation," Tappi Journal 78, No. 11, November 1995.

8. H. Dahl, H. Holik, and E. Weisshuhn, "The influence of headbox flow conditions on paper properties and their constancy," Tappi Journal, pp. 93-98, February 1988.

9. T. A. Vuori and C. L. Smith, "Three dimensional imaging system with structured lighting and practical constraints," Journal of Electronic Imaging 6, No. 1, pp. 140-144, January 1997.

10. H. Kaplan, "Structured light finds a home in machine vision," Photonics Spectra, January 1994.

11. F. W. DePiero and M. M. Trivedi, "3-D Computer Vision Using Structured Light: Design, Calibration and Implementation Issues," Advances in Computers 43, 1996.

12. M. Johannesson, "Sheet-of-light Range Imaging," Linköping Studies in Science and Technology, Dissertation No. 399, SIMD Architectures for Range and Radar Imaging, Linköping, Sweden, 1995. 